ISSN 0103-5150

Fisioter. Mov., Curitiba, v. 24, n. 1, p. 57-64, jan./mar. 2011 Licenciado sob uma Licença Creative Commons

\title{
Análise dos efeitos da cinesioterapia e da hidrocinesioterapia sobre a qualidade de vida de pacientes com fibromialgia - um ensaio clínico randomizado
}

\author{
Analysis of effects of kinesiotherapy and hydrokinesiotherapy on \\ the quality of life of patients with fibromyalgia - a randomized \\ clinical trial
}

\author{
Celina Dani Hecker ${ }^{[a]}$, Cyntia Melo ${ }^{[a]}$, Shaiane da Silva Tomazoni ${ }^{[b]}$, \\ Rodrigo Álvaro Brandão Lopes Martins ${ }^{[\mathrm{c}]}$, Ernesto Cesar Pinto Leal Junior ${ }^{[\mathrm{d}]}$
}

[a] Fisioterapeuta, Universidade de Caxias do Sul (UCS), Caxias do Sul, RS - Brasil.

[b] Fisioterapeuta, Mestranda em Farmacologia, Laboratório de Farmacologia e Terapêutica Experimental, Departamento de Farmacologia, Instituto de Ciências Biomédicas (ICB), Universidade de São Paulo (USP), São Paulo, SP - Brasil.

[c] Biólogo, PhD, chefe do Laboratório de Farmacologia e Terapêutica Experimental, Departamento de Farmacologia, Instituto de Ciências Biomédicas (ICB), Universidade de São Paulo (USP), São Paulo, SP - Brasil.

[d] Fisioterapeuta, PhD, Programa de Pós-Graduação em Ciências da Reabilitação, Universidade Nove de Julho (UNINOVE), São Paulo, SP - Brasil, e-mail: ernesto.leal.junior@gmail.com

\section{Resumo}

Objetivo: Verificar e comparar os efeitos da hidrocinesioterapia e da cinesioterapia na qualidade de vida de pacientes portadoras de fibromialgia. Materiais e métodos: Foi realizado um ensaio clínico randomizado, cego, para os avaliadores. Para avaliar a qualidade de vida, utilizou-se o questionário genérico SF-36 a fim de analisar os aspectos físicos e psicológicos em 24 pacientes do sexo feminino, portadoras de fibromialgia, com idade de 30 a 55 anos, alocadas aleatoriamente em dois grupos: hidrocinesioterapia e cinesioterapia. Durante um período de 23 semanas, as pacientes foram submetidas a alongamentos e exercícios aeróbios de baixa intensidade com duração de uma hora cada sessão (em ambos os grupos). Resultados: Observouse melhora estatisticamente significante $(\mathrm{p}<0,05)$ na maior parte dos aspectos abordados pelo SF-36 em ambos os grupos. Conclusão: A hidrocinesioterapia promoveu um relaxamento muscular favorável para a melhora da qualidade de vida, porém, não foi o fator principal para amenizar os efeitos da patologia, uma vez que a cinesioterapia também proporcionou efeitos benéficos para a promoção do bem-estar dessas pacientes. Portanto, os alongamentos e os exercícios aeróbios de baixa intensidade empregados em ambos 
os protocolos são os prováveis responsáveis pelos efeitos benéficos observados nas duas modalidades terapêuticas analisadas.

Palavras-chave: Fibromialgia. Qualidade de vida. Hidroterapia. Cinesioterapia.

\section{Abstract}

Objective: To verify and compare the effects of hydrokinesiotherapy and kinesiotherapy in the quality of life of patients with fibromyalgia. Materials and methods: It was realized a randomized clinical trial, blinded, to observers. To evaluate the quality of life, it was used the generic questionnaire SF- 36 in order to analyze the physical and psychological aspects, in 24 female patients with fibromyalgia, between 30 and 55 years old, placed aleatorialy in two groups: hydrokinesiotherapy and kinesiotherapy. During a period of 23 weeks, the patients were submitted to stretching and aerobic exercises of low intensity with one hour of duration each section (in both groups). Results: It was observed statistically significant increase $(p<0,05)$ in the majority of the aspects of SF-36 in both groups. Conclusion: The hydrokinesiotherapy provides a favorable muscle relaxing to improve the quality of life, however it is not the main factor to decrease the effects of the pathology, since the kinesiotherapy also provided beneficent effects to promote the well being of these patients. Therefore, stretching and aerobic exercises of low intensity employed in both protocols are the probable responsibles by positive effects observed in both therapeutic modalities analysed.

Keywords: Fibromyalgia. Quality of life. Hydrotherapy. Kinesiotherapy.

\section{Introdução}

A fibromialgia é uma síndrome complexa caracterizada por dores difusas e crônicas que limitam as atividades de vida diária. Frequentemente, associase à incapacidade funcional, à depressão, à ansiedade, ao sono não reparador, à fadiga, ao déficit de memória, à cefaleia, à constipação ou à diarreia $(1,2)$. A maior parte dos pacientes acometidos (88\%) são mulheres, sendo mais comum na faixa etária entre 40 e 50 anos. Estima-se que a prevalência na população geral é de $2 \%$ a $5 \%$ (3-7).

As opções terapêuticas para o tratamento da fibromialgia incluem medicações para reduzir a dor e melhorar o sono, programas de exercícios para fortalecer a musculatura e melhorar a aptidão cardiovascular, técnicas de relaxamento para combater a tensão muscular e programas educativos para ajudar a entender e manejar a fibromialgia $(8,9)$. A fisioterapia tem um importante papel na melhora do controle da dor e no aumento ou na manutenção das habilidades funcionais do paciente em casa ou no trabalho (10).

A cinesioterapia compreende dois grandes objetivos da fisioterapia no tratamento da fibromialgia: exercitar os músculos doloridos com exercícios de alongamento e melhorar as condições cardiovasculares com exercícios aeróbios (10). Exercícios físicos de baixa intensidade são considerados mais eficazes na diminuição do impacto da doença e na qualidade de vida desses pacientes (11).

A hidroterapia consiste em um recurso terapêutico abrangente que utiliza os exercícios aquáticos para ajudar na reabilitação de várias patologias (12). Esse recurso proporciona grande alívio dos sintomas da fibromialgia, pois os movimentos na água são lentos e dão suporte às estruturas corporais permitindo maior mobilidade e, consequentemente, alongamentos mais eficientes (13). Existem também os benefícios da imersão do paciente em água aquecida, que deve estar em torno de $30{ }^{\circ} \mathrm{C}$ a $34^{\circ} \mathrm{C}$, favorecendo o relaxamento muscular e diminuindo a dor e a rigidez (14).

A fibromialgia afeta negativamente na qualidade de vida dos pacientes. 0 impacto global envolve aspectos pessoais, profissionais, familiares e sociais, correlacionando-se fortemente com a intensidade da dor, fadiga e decréscimo da capacidade funcional (15).

Qualidade de vida é a "percepção do indivíduo de sua posição na vida, no contexto da cultura e sistema de valores nos quais ele vive em relação aos seus objetivos, expectativas, padrões e preocupações" (16), por isso compreende uma medida de resultado importante no estudo de desordens reumáticas. 
A maior parte das pesquisas sobre qualidade de vida é feita usando instrumentos validados, como o questionário de avaliação SF-36, que avalia aspectos relativos à função, à disfunção e ao desconforto físico e emocional $(17,18)$.

Encontrar alternativas efetivas de tratamento que minimizem o impacto da fibromialgia sobre a qualidade de vida dos pacientes é fundamental para o sistema de saúde. Ao abordar a questão das limitações funcionais decorrentes da fibromialgia e suas consequências sobre a qualidade de vida, torna-se necessário ampliar a perspectiva do impacto dos sintomas, pois as áreas afetadas se tornam tão importantes quanto a doença em si (2).

Muitas vezes, os pacientes acometidos pela patologia não possuem tempo disponível para duas ou mais sessões de tratamento semanais e, em alguns casos, os pacientes também não possuem acesso ao tratamento em piscinas terapêuticas aquecidas.

Sendo assim, o objetivo do presente estudo consistiu em investigar os efeitos de um protocolo de tratamento realizado uma vez por semana, utilizando a hidrocinesioterapia ou a cinesioterapia como recursos terapêuticos sobre a qualidade de vida de mulheres portadoras de fibromialgia, comparando a eficácia dos dois métodos terapêuticos utilizados.

\section{Materiais e métodos}

Foi realizado um ensaio clínico randomizado, cego, para os avaliadores, em que os pacientes foram alocados aleatoriamente em dois grupos de tratamento: hidrocinesioterapia e cinesioterapia. Ambos receberam os atendimentos em grupo.

Cada sessão de tratamento teve duração de 60 minutos, sendo realizada uma vez por semana por um período de 23 semanas, com controle dos sinais vitais no início e ao fim de cada sessão de tratamento e questionamento sobre o uso de medicamentos por parte das pacientes.

\section{Amostra}

Foram estudadas 24 pacientes do sexo feminino, com idade entre 30 e 55 anos, apresentando diagnóstico clínico de fibromialgia, alocadas aleatoriamente nos respectivos grupos de acordo com o tratamento recebido: a) grupo hidrocinesioterapia: composto por 12 pacientes do sexo feminino, com idade média de 47,5 anos $( \pm 5,78)$, tempo médio de sintomatologia de 7,0 anos $( \pm 4,46)$ e tempo de diagnóstico da patologia de 3,0 anos $( \pm 2,92)$;

b) grupo cinesioterapia: também composto por 12 pacientes do sexo feminino, com idade média de 45,3 anos $( \pm 8,02)$, tempo médio de sintomatologia de 6,9 anos $( \pm 1,83)$ e tempo de diagnóstico da patologia de 4,5 anos $( \pm 1,83)$.

\section{Critérios de inclusão}

Foram incluídas no estudo pacientes do sexo feminino, com idade entre 30 e 55 anos, com fibromialgia diagnosticada há pelo menos dois anos, sem o diagnóstico de doenças associadas, não praticantes de atividade física regular e que não estivessem fazendo uso de medicamentos.

\section{Critérios de exclusão}

Foram excluídas do estudo as pacientes com idade inferior a 30 e superior a 55 anos, pacientes que apresentassem alguma outra patologia que impedisse o tratamento e as voluntárias com frequência inferior a 75\% nos atendimentos para ambos os grupos. Também foram excluídas do estudo as que estavam fazendo uso de qualquer tipo de medicamento, bem como aquelas que passaram a fazer uso de medicamentos durante a realização do estudo.

\section{Instrumentos}

Foi utilizado o questionário genérico SF-36, elaborado para avaliar a qualidade de vida, tendo sido validado no Brasil por Ciconelli et al. (17). Esse questionário é composto de 11 campos de avaliação, abordando fatores físicos e psicológicos da paciente. As questões são agrupadas em oito domínios, os quais abordam aspectos físicos, capacidade funcional, aspectos emocionais, saúde mental, estado geral de saúde, vitalidade, dor e aspectos sociais. Cada domínio varia de 0 a 100 e quanto maior a aproximação de 100 melhor é a qualidade de vida. Também foram utilizados para as sessões de 
tratamento: estetoscópio, esfigmomanômetro, bastões de madeira, pranchas de treinamento sensóriomotor e steps.

\section{Procedimentos}

Foram selecionados aleatoriamente 30 prontuários de ex-pacientes, portadoras de fibromialgia, nos arquivos da Clínica de Fisioterapia da Universidade de Caxias do Sul (UCS), com idade entre 30 e 55 anos. Realizou-se contato inicial por telefone com as pacientes que preenchiam os critérios de inclusão, totalizando amostra de 24 pacientes. Posteriormente, todas tiveram suas avaliações agendadas para a Clínica de Fisioterapia da UCS.

Primeiramente, houve breve apresentação e explicação sobre a importância do trabalho diante da patologia apresentada. Sete dias antes da primeira intervenção, realizou-se uma avaliação individual, em que constavam os seguintes itens: nome, data de nascimento, idade, estado civil, escolaridade, profissão, massa corporal, estatura, uso de medicamentos, prática de atividade física regular, outras doenças, tempo de sintomatologia, tempo de diagnóstico e tempo de tratamento. Nesse momento, aplicou-se também o questionário genérico de qualidade de vida SF-36 e as pacientes tiveram liberdade para esclarecer possíveis dúvidas sobre o questionário.

0 tratamento de hidrocinesioterapia consistiu em: exercícios de alongamento muscular ao início da sessão de tratamento (com duração de 60 segundos cada exercício) para os principais grupos musculares de membros inferiores, membros superiores, tronco e pescoço (15 minutos no total); exercícios aeróbios de baixa intensidade, como caminhadas para frente, para trás e lateralmente $(5$ minutos cada - 15 minutos no total), e movimentação ativa sem carga dos membros inferiores, membros superiores, tronco e pescoço (15 minutos no total); e finalizando com os mesmos exercícios de alongamento muscular realizados no início da sessão (15 minutos no total). A temperatura da água permaneceu entre $32{ }^{\circ} \mathrm{C} \mathrm{e} 34^{\circ} \mathrm{C}$.

0 protocolo de cinesioterapia consistiu exatamente nos mesmos exercícios aeróbios de baixa intensidade e de alongamento muscular descritos anteriormente, porém, realizados fora do ambiente aquático.

Durante o período de realização do estudo, as pacientes não foram submetidas a nenhum outro tipo de tratamento físico ou farmacológico. Tais pacientes foram reavaliadas sete dias após a $23^{\mathfrak{a}}$ terapia, seguindo os mesmos parâmetros e procedimentos descritos anteriormente.

\section{Aspectos éticos}

Previamente ao início, as pacientes tomaram conhecimento dos procedimentos do estudo e assinaram um termo de consentimento livre e esclarecido em que ficou documentada e registrada sua participação.

O trabalho foi submetido e aprovado pelo Comitê de Ética em Pesquisa da Universidade de Caxias do Sul (Parecer n. 31/2006) conforme determina a resolução 196/96 do Conselho Nacional de Saúde (CNS).

\section{Análise dos dados}

Os dados foram expressos pela média dos valores obtidos pelos grupos estudados para cada aspecto avaliado pelo SF-36 e analisados quanto à sua significância estatística pelo teste $t$ de student pareado para as análises intragrupo e pelo teste $t$ de student não pareado para as análises intergrupos. Para ambas as análises foi estabelecido o valor de $\mathrm{p}<0,05$.

\section{Resultados}

Nas comparações entre os dois grupos estudados, não foram verificadas diferenças estatisticamente significantes $(p>0,05)$ nas avaliações realizadas antes do início do estudo, e após o seu término, para nenhum dos oito aspectos analisados pelo SF-36.

Já nas análises intragrupos, ambas as medidas terapêuticas mostraram-se eficazes $(\mathrm{p}<0,05)$ no incremento da maior parte dos aspectos abordados pelo SF-36, conforme demonstrado na Tabela 1.

\section{Discussão}

A qualidade de vida abrange aspectos físicos, sociais e emocionais (17). Neste estudo foram analisados os aspectos referentes à qualidade de vida utilizando o questionário validado SF-36. Este foi aplicado em um período pré e pós-tratamento de 
Tabela 1 - Média dos valores obtidos nas avaliações pré e pós-tratamento dos aspectos analisados pelo SF-36

\begin{tabular}{|c|c|c|c|c|}
\hline & \multicolumn{2}{|c|}{ Hidrocinesioterapia } & \multicolumn{2}{|c|}{ Cinesioterapia } \\
\hline & Pré & Pós & Pré & Pós \\
\hline Capacidade funcional & $26,25( \pm 25,77)$ & $43,33^{\star}( \pm 16,28)$ & $24,17( \pm 27,54)$ & $41,67^{*}( \pm 20,04)$ \\
\hline Aspectos físicos & $12,50( \pm 22,61)$ & $20,83 \quad( \pm 33,43)$ & $10,42( \pm 29,11)$ & $27,75^{\star}( \pm 31,04)$ \\
\hline Aspectos emocionais & $16,58( \pm 30,10)$ & $44,50 *( \pm 35,92)$ & $16,67( \pm 38,92)$ & $26,58 \quad( \pm 27,83)$ \\
\hline Dor & $20,58( \pm 15,42)$ & $34,25^{\star}( \pm 11,05)$ & $22,75( \pm 16,26)$ & $40,58^{*}( \pm 16,09)$ \\
\hline Estado geral de saúde & $34,50( \pm 14,63)$ & $41,33 \quad( \pm 14,93)$ & $29,25( \pm 28,79)$ & $39,58 \quad( \pm 16,25)$ \\
\hline Vitalidade & $28,75( \pm 29,01)$ & $38,75 \quad( \pm 14,64)$ & $25,42( \pm 26,15)$ & $42,08 \quad( \pm 14,37)$ \\
\hline Aspectos sociais & $35,58( \pm 32,69)$ & $61,67^{\star}( \pm 22,23)$ & $28,33( \pm 27,36)$ & $50,58^{*}( \pm 16,23)$ \\
\hline Saúde mental & $34,33( \pm 24,68)$ & $50,67^{\star}( \pm 15,19)$ & $28,33( \pm 23,78)$ & $45,00 *( \pm 16,72)$ \\
\hline
\end{tabular}

Legenda: * = diferença estatisticamente significante $(p<0,05)$ entre avaliação pré e pós-tratamento (avaliação intragrupo).

dois grupos, um recebendo tratamento com hidrocinesioterapia e o outro com cinesioterapia.

Nas comparações entre os dois grupos, não foram verificadas diferenças estatisticamente significantes ( $\mathrm{p}>0,05)$, nos períodos pré e pós-tratamento, para nenhum dos oito aspectos analisados. Nosso resultado confere com o resultado de Vitorino et al. (18), que analisaram por meio do SF-36 a qualidade de vida em pacientes com fibromialgia e também não observaram diferença entre o tratamento com hidroterapia e fisioterapia convencional.

Na capacidade funcional, ambos os grupos apresentaram melhora estatisticamente significante $(\mathrm{p}<$ $0,05)$ entre o período pré e pós-tratamento. Esses dados concordam com os de outros estudos que evidenciam os benefícios adicionais a longo e curto prazo dos exercícios aquáticos $(19,20)$. Os efeitos físicos, fisiológicos e cinesiológicos advindos da imersão do corpo auxiliam na reeducação musculoesquelética, visando ao restabelecimento da saúde e incluindo o alívio da sintomatologia para manutenção do exercício (21).

A melhora obtida com o tratamento de cinesioterapia pode estar relacionada ao bem-estar geral promovido pela liberação de endorfinas no sistema nervoso central por meio do alongamento muscular e dos exercícios de baixa intensidade (22).

Gashu et al. (23) verificaram um papel importante na sintomatologia por meio do alongamento muscular e da massoterapia. Para McCain et al. (24), a prática de exercícios aeróbios de baixa intensidade, como a caminhada, tem demonstrado resultados promissores na melhora da qualidade de vida. Esse autor observou discreta superioridade de efeitos benéficos do condicionamento aeróbio em relação ao alongamento muscular em uma comparação entre as duas técnicas.

Referente aos aspectos físicos, somente o grupo tratado com cinesioterapia obteve melhora estatisticamente significante $(\mathrm{p}<0,05)$. Isso pode estar relacionado aos efeitos do alongamento na recuperação da amplitude de movimento e da mobilidade articular (25), agindo positivamente sobre a sintomatologia da fibromialgia e facilitando a execução das atividades de vida diária $(21,26)$.

Para Valim (27) e Sabbag et al. (11), o condicionamento aeróbio com exercícios de baixa intensidade seria superior ao alongamento na melhora dos aspectos físicos. Gowans et al. (28) referiram que 23 semanas de exercícios aeróbios supervisionados produziram melhoras significativas na função e no modo físico de pacientes com fibromialgia por meio de uma avaliação realizada 12 meses após o término da intervenção.

Quanto aos aspectos emocionais, somente o grupo da hidrocinesioterapia apresentou melhora estatisticamente significante $(\mathrm{p}<0,05)$ quando comparados os resultados pré e pós-tratamento. A significante melhora se deve, provavelmente, ao fato da água facilitar a performance dos movimentos e promover relaxamento muscular (14), uma vez que a flutuação contrapõe-se à gravidade aliviando o peso corporal e, consequentemente, diminuindo a força de compressão sobre as articulações e o trabalho 
muscular (13). Não existem estudos que comprovem a eficácia plena da hidrocinesioterapia diante dos aspectos emocionais. Acreditamos que, com a melhora da sintomatologia promovida pelos efeitos dessa técnica, as pacientes se sintam mais dispostas e menos deprimidas ou ansiosas.

Neste estudo, o grupo que recebeu tratamento com cinesioterapia não apresentou melhora estatisticamente significante para os aspectos emocionais. McCain et al. (24) e Buckelew et al. (29) avaliaram o impacto dos exercícios cinesioterapêuticos sobre os aspectos emocionais e verificaram melhora. Segundo estudos de Nichols et al. (30), esses aspectos modificaram-se com o condicionamento aeróbio, mas não com técnicas de alongamento.

No aspecto dor, a melhora foi estatisticamente significante para os dois grupos $(p<0,05)$. Podemos atribuir esse resultado ao fato de que durante a imersão ocorre aumento da circulação e redução dos espasmos, além disso, os estímulos sensoriais competem com os dolorosos, interrompendo o ciclo da dor (13). Nesse sentido, o meio aquático favorece a execução de atividades rotineiras pelo alívio dos sintomas dolorosos (31).

Já por meio da cinesioterapia, o alongamento interfere na flexibilidade, relaxando os músculos que estão contraídos e rígidos, diminuindo a dor (26, 32 ). Outros autores evidenciam que a atividade física poderia modular a dor, sendo capaz de aumentar a tolerância a esse estímulo (27, 33-36).

Mesmo diante de todos os efeitos terapêuticos do ambiente aquático (relaxamento muscular, diminuição da tensão muscular e da dor) $(13,37)$ e da terapia aplicada em solo, que proporciona ganho de condicionamento físico, aumento da flexibilidade, diminuição da dor e da fadiga muscular $(10,27)$, não foram observadas diferenças estatisticamente significantes nas avaliações pré e pós-tratamento para os grupos tratados com hidrocinesioterapia e cinesioterapia ( $p>0,05)$ para os aspectos estado geral de saúde e vitalidade. Talvez fosse necessário maior tempo de intervenção para que se verificassem resultados significantes nesses aspectos.

Nos aspectos sociais ambos os grupos apresentaram diferença estatisticamente significante $(\mathrm{p}<0,05)$. Sugere-se o fato de que a diminuição da dor ocasionada pelas duas técnicas refletiu positivamente nesse aspecto, uma vez que a dor limita as atividades diárias e incapacita o paciente de realizar tarefas em grupos pelo desconforto dessa sintomatologia (8).
No que se refere à Saúde mental houve melhora e a diferença foi estatisticamente significante para os dois grupos $(\mathrm{p}<0,05)$. Sugere-se que o exercício é capaz de interferir positivamente no estado mental, melhorando a autoestima e diminuindo a depressão das pacientes (38). Isso foi verificado no decorrer deste estudo, tanto nas pacientes que receberam tratamento de cinesioterapia quanto nas que receberam tratamento de hidrocinesioterapia. Houve um incremento significativo na saúde mental, provavelmente por causa do bem-estar geral promovido pelo exercício e convívio com pessoas apresentando a mesma patologia, sendo tais resultados também evidenciados por Heriksson et al. (39).

Torna-se fundamental salientar que as técnicas empregadas como medidas de tratamento, tanto pela hidrocinesioterapia quanto pela cinesioterapia, diferiram basicamente entre si com relação à imersão em água aquecida. Ambas foram compostas por exercícios aeróbios de baixa intensidade e alongamentos musculares.

Os resultados apresentados pelos dois grupos demonstraram grande eficácia no incremento da qualidade de vida das pacientes e não evidenciaram diferença entre os dois métodos empregados. Nesse sentido, conclui-se que um programa de tratamento realizado uma vez por semana, contendo exercícios aeróbios de baixa intensidade e exercícios de alongamento muscular, sendo ou não realizados no ambiente aquático, é um recurso indispensável para o tratamento de pacientes acometidas por fibromialgia, uma vez que permite melhora em praticamente todos os aspectos referentes à qualidade de vida.

Sendo assim, os exercícios cinesioterapêuticos podem ser aplicados em grupos de pacientes portadoras da patologia, inclusive em Unidades Básicas de Saúde (UBSs), promovendo melhor qualidade de vida para as pacientes e tendo baixo custo financeiro em sua aplicação.

\section{Referências}

1. Wolfe F. Fibromyalgia: the clinical syndrome. Rheum Dis Clin North Am. 1989;15(1):1-18.

2. Ruof J, Hulsemann J, Stucki G. Evaluation of costs in rheumatic diseases: a literature review. Curr Opin Rheumatol. 1999;11(2):104-9. 
3. Wolfe F, Russell IJ, Vipraio G, Ross K, Anderson J. Serotonin levels pain threshold, and fibromyalgia symptoms in the general population. J Rheumatol. 1997;24(3):555-9.

4. Wolfe F, Ross K, Anderson J, Russell IJ, Hebert L. The prevalence and characteristics of fibromyalgia in the general population. Arthritis Rheum. 1995; 38(1):19-28.

5. Mäkelä M, Heliövaara M. Prevalence of primary fibromyalgia in the Finnish population. BMJ. 1991; 303(6796):216-9.

6. McCain GA. A clinical overview of the fibromyalgia syndrome: a comparison with rheumatoid arthritis. J Musculoskel Pain. 1996;4:9-34.

7. Hartz A, Kirchdoefer E. Undetected fibrositis in primary care practice. J Fam Pract. 1987;25(4):365-9.

8. Jentoft ES, Kvalvik AG, Mengshoel AM. Effects of poolbased and land-based aerobic exercise on women with fibromyalgia/chronic widespread muscle pain. Arthritis Rheum. 2001;45(1):42-7.

9. Goldenberg DL, Mossey CJ, Schimid CH. A model to assess severity and impact of fibromyalgia. J Rheumatol. 2001;45(1):42-7.

10. Clark SR, Jones KD, Burckhardt CS, Bennett R. Exercise for patients with fibromyalgia: risks versus benefits. Curr Rheumatol Rep. 2001;3(2):135-46.

11. Sabbag LMS, Dourado MP, Yasbek Jr P, Novo NF, Kaziyama HHS, Miyazaki MH, et al. Estudo ergométrico evolutivo de portadoras de fibromialgia primária em programa de treinamento cardiovascular supervisionado. Acta Fisiátrica. 2000;7(1):29-34.

12. Becker BE. Princípios físicos da água. In: Ruoti RG, Morris DM, Cole AJ. Reabilitação aquática. São Paulo: Manole; 2000. p. 17-27.

13. Bates A, Hanson N. Aquatic exercise therapy. Philadelphia, PA: W. B. Saunders; 1998.

14. Mannerkorpi K, Iversen MD. Physical exercise in fibromyalgia and related syndromes. Best Pract Res Clin Rheumatol. 2003;17:629-47.

15. Crook J, Moldofsky H, Shannon H. Determinants of disability after a work related musculoskeletal injury. J Rheumatol. 1998;25(8):1570-7.
16. Neumann L, Buskila D. Quality of life and physical functioning of relatives of fibromyalgia patients. Semin Arthritis Rheum. 1997;26(6):834-9.

17. Ciconelli RM, Ferraz MB, Santos W. Tradução para a língua portuguesa e validação do questionário genérico de avaliação de qualidade de vida SF-36 (Brasil SF-36). Rev Bras Reumatol. 1998;39(3):143-50.

18. Vitorino DF, Carvalho LB, Prado GF. Hydrotherapy and conventional physiotherapy improve total sleep time and quality of life of fibromyalgia patients: randomized clinical trial. Sleep Med. 2006;7(3):293-6.

19. Mannerkorpi K, Ahlmén M, Ekdahl C. Six- and 24-month follow-up of pool exercise therapy and education for patients with fibromyalgia. Scand J Rheumatol. 2002;31(5):306-10.

20. Altan L, Bingöl U, Aykaç M, Koç Z, Yurtkuran M. Investigation of the effects of pool-based exercise on fibromyalgia syndrome. Rheumatol Int. 2004;24(5): 272-7.

21. Marques AP, Furtado LLM, Cossermeli W. Alongamento muscular em pacientes com fibromialgia a partir de um trabalho de reeducação postural global. Rev Bras Reumatol. 1994;34(5):232-4.

22. de Mendonça LLF, Marques AP, Matsutani LA, Ferreira EAG. Exercícios de alongamento para pacientes com fibromialgia. Rev Bras Reumatol. 2002; 42(1):49-50.

23. Gashu BM, Marques AP. Efeito da estimulação elétrica transcutânea sobre os tender points dos pacientes fibromiálgicos: estudo preliminar. Rev Bras Fisioter. 1997;8(2):57-62.

24. McCain GA, Cameron R, Kennedy JC. The problem of longterm disability payments and litigation in primary fibromyalgia: the Canadian perspective. J Rheumatol. 1989;19(Suppl.):174-6.

25. Helfenstein M, Feldman D. Síndrome da fibromialgia: características clínicas e associações com outras síndromes disfuncionais. Rev Bras Reumatol. 2002; 42(1):8-14.

26. Atra E, Pollak DF, Martinez JE. Fibromialgia: etiopatogenia e terapêutica. Rev Bras Reumatol. 1993; 33(2):66-72.

27. Valim V. Benefícios dos exercícios físicos na fibromialgia. Rev Bras Reumatol. 2006;46(1):49-55. 
28. Gowans SE, Dehueck A, Voss S, Silaj A, Abbey SE. Sixmonth and one-year followup of 23 weeks of aerobic exercise for individuals with fibromyalgia. Arthritis Rheum. 2004;51(6):890-8.

29. Buckelew SP, Conway R, Parker J, Deuser WE, Read J, Witty TE. Biofeedback/relaxation training and exercise interventions for fibromyalgia: a prospective trial. Arthritis Care Res. 1998;11(3):196-209.

30. Nichols DS, Glenn TM. Effects of aerobic exercise on pain perception, affect, and level of disability in individuals with fibromyalgia. Phys Ther. 1994; $74(4): 327-32$.

31. Martinez JE. Avaliação da qualidade de vida de pacientes com fibromialgia através do "Medical Outcome Survey 36 Item Short Form Study". Rev Bras Reumatol. 1999;39(6):312-6.

32. Elert J, Kendall SA, Larsson B, Mansson B, Gerdle B. Chronic pain and difficulty in relaxing postural muscles in patients with fibromyalgia and chronic whiplash associated disorders. J Rheumatol. 2001; 28(6):1361-8.

33. Moldofsky H. Sleep and fibrositis syndrome. Rheum Dis Clin North Am. 1989;15(1):91-103.

34. Haier RJ, Quaid K, Mills JC. Naloxone alters pain perception after jogging. Psychiatry Res. 1981;5(2):231-2.
35. Granges G, Zilko P, Littlejohn GO. Fibromyalgia syndrome: assessment of the severity of the condition 2 years after diagnosis. J Rheumatol. 1994;21(3):523-9.

36. Yunus MB. Psychological aspects of fibromyalgia syndrome: a component of the dysfunctional spectrum syndrome. Baillieres Clin Rheumatol. 1994; 8(4):811-37.

37. Kisner C, Colby LA. Exercícios terapêuticos, fundamentos e técnicas. 3a ed. São Paulo: Manole; 1998.

38. Hammett VB. Psychological changes with physical fitness training. Can Med Assoc J. 1967;96(12):764-9.

39. Henriksson C, Gundmark I, Bengtsson A, Ek AC. Living with fibromyalgia. Consequences for everyday life. Clin J Pain. 1992;8(2):138-44.

Recebido: 24/04/2009 Received: 04/24/2009 Aprovado: 05/07/2010 Approved: 07/05/2010 